\title{
Electron Spin-Phonon Relaxation in Quantum Dots
}

\author{
A. M. Alcalde and G. E. Marques \\ Departamento de Física, Universidade Federal do São Carlos \\ Caixa Postal 676, 13565-905 São Carlos, SP, Brazil
}

Received on 31 March, 2003

\begin{abstract}
We calculate the spin relaxation rates in parabolic quantum dots due to the phonon modulation of the spin-orbit interaction in presence of an external magnetic field. Both, deformation potential (DP) and piezoelectric (PE) electron-phonon couplings are included in the Pavlov-Firsov spin-phonon Hamiltonian. We demonstrate that the spin relaxation rates are particularly sensitive with the Landé $g$-factor, and that for InAs dots the DP can be necessarily considered in the spin relaxation analysis. Low-temperature $(T \sim 0)$ relaxation rates are found to be small and to depend strongly on size, $g$-factor, and on magnetic field.
\end{abstract}

\section{Introduction}

The ability to manipulate and control processes that involve transitions between spin states is, at the moment, of extreme importance due to the recent applications in quantum computation and quantum communication. Quantum dots of diverse geometries are serious candidates for implementation of semiconductor quantum communication devices because the electronic, magnetic and optical properties can be controlled through the modern grown and nanofabrication techniques. The spin dephasing time is important because it sets the length-scale on which coherent physics can be observed. It is therefore important to understand the origin of decoherence so that ultimately it may be reduced or controlled. At the moment remains in discussion, which between these processes is dominant in semiconductors especially in low dimensional systems. Some experimental results have showed good agreement with the theoretical predictions in 2-D systems [1], but in general, the identification of the processes through direct comparison with the experimental results can become a formidable task. This problem is more critical in quantum dots, since few experimental results exist and the theoretical discussion of the spin relaxation mechanisms is still an open subject. Khaetskii and Nazarov [2, 3] studied different processes of spin-flip in GaAs quantum dots. In general, was showed that the quantum confinement produces a strong reduction of the relaxation rates, with obtained magnitudes of the order of $10^{1}-10^{4}\left(\mathrm{~s}^{-1}\right)$. Recently, Woods et al. [4] have studied the effects of the phonon coupling due to interface motion, small and strongly size dependent rates have been found by the authors. In this work we calculate the spin-flip transition rates, considering the phonon modulation of the spin-orbit interaction. We will also study the effects of the Landé $g$-factor and the contributions of the diverse coupling processes with acoustic phonons.

\section{Theory}

Experimental measurements and numerical calculations [see Ref. [5] and references therein] indicate that in lens-shaped quasi-two dimensional self-assembled quantum dots the bound states of both electrons and valence-band holes can be understood assuming an effective parabolic potential $V(\rho)=\frac{1}{2} m \omega_{0}^{2} \rho^{2}$, where $\hbar \omega_{0}$ is the characteristic confinement energy, and $\rho$ is the radial coordinate. By using a oneband effective mass approximation and considering the presence of an external magnetic field $B$ applied normal to plane of the dot, we write the electron wave functions as

$$
f_{n, l, \sigma}=\left[\frac{n !}{\pi(n+|l|) !}\right]^{\frac{1}{2}} \frac{\rho^{|l|}}{a^{|l|+1}} e^{-\frac{\rho^{2}}{2 a^{2}}} e^{i l \varphi} L_{n}^{|l|}\left(\frac{\rho^{2}}{a^{2}}\right) \chi(\sigma) .
$$

In the above expression $L_{n}^{|m|}$ denotes the Laguerre polynomials, $n$ is the principal quantum number, $l$ is the azimuthal quantum number and $\chi(\sigma)$ is the spin wave function of spin variable $\sigma$. The corresponding eigenenergies are $E_{n, l, \sigma}=(2 n+|l|+1) \hbar \Omega+(l / 2) \hbar \omega_{c}+(\sigma / 2) g \mu_{B} B$, where $\Omega=\left(\omega_{0}^{2}+\omega_{c}^{2} / 4\right)^{1 / 2}, \mu_{B}$ is the Bohr magneton, $a=(\hbar / m \Omega)^{1 / 2}$ is the effective length, $\omega_{c}=e B / m$. The Landé $g$-factor and the effective mass $m$ are expressed in second-order $\mathbf{k} \cdot \mathbf{p}$ perturbation $[6,7]$

$$
\begin{aligned}
& g=2-\frac{4 m_{0} P^{2}}{3 \hbar^{2}} \frac{\Delta}{\left(E_{g}+E\right)\left[\left(E_{g}+E\right)+\Delta\right]}, \\
& \frac{1}{m}=\frac{1}{m_{0}}+\frac{2 P^{2}}{3 \hbar^{2}} \frac{3\left(E_{g}+E\right)+2 \Delta}{\left(E_{g}+E\right)\left[\left(E_{g}+E\right)+\Delta\right]} .
\end{aligned}
$$

Here $g_{0}$ is the free-electron $g$-factor, $\Delta$ is the spin-orbit splitting, $P$ represent the interband matrix element, and $\mathcal{E}$ is the fundamental gap renormalized by the confinement. In InAs the value of $\Delta$ is comparable with de fundamental gap, thus we can expect significant variations of the electron $g$-factor with the size parameters. 
For an assisted acoustic-phonon spin-flip process, the matrix element $M$ of the electron spin-flip between an initial state $|n l\rangle$ and spin $\sigma_{z}=+1(\uparrow)$ and a final state $\left|n^{\prime} l^{\prime}\right\rangle$ and spin $\sigma_{z}=-1(\downarrow)$ with emission of a phonon of momentum q and energy $\hbar v q$, can be obtained from the Pavlov-Firsov spin-phonon Hamiltonian $[8,9,10]$ as

$$
\begin{aligned}
M_{n l \uparrow \rightarrow n^{\prime} l^{\prime} \downarrow}= & d(q)\left(\frac{\hbar}{\rho_{M} V v q}\right)^{1 / 2} \\
& \chi_{z}(\uparrow)\left(\begin{array}{cc}
0 & \hat{\mathbf{n}}^{-} \times \hat{\mathbf{e}}_{\mathbf{q}} \\
\hat{\mathbf{n}}^{+} \times \hat{\mathbf{e}}_{\mathbf{q}} & 0
\end{array}\right) \chi_{z}(\downarrow) . \\
& \int d^{3} \mathbf{r} f_{n^{\prime} l^{\prime}} e^{-i \mathbf{q} \cdot \mathbf{r}}\left(\frac{\mathbf{p}}{\hbar}+\frac{e \mathbf{A}}{\hbar c}+\mathbf{q}\right) f_{n l},
\end{aligned}
$$

where $\chi_{z}$ are the spin wavefunctions quantized along the $z$ axis, $f_{n l}=\langle\mathbf{r} \mid \mathbf{n}, \mathbf{l}\rangle$ is the electron envelope wavefunction. The magnetic vector potential $\mathbf{A}$ is obtained in the symmetric gauge considering that the orientation of $\mathbf{B}$ coincide with the $z$-axis. $\hat{\mathbf{n}}^{ \pm}=\hat{\mathbf{x}} \pm \hat{\mathbf{y}}, \mathbf{p}$ is the momentum operator, $\hat{\mathbf{e}}_{\mathbf{q}}$ is the phonon polarization vector, $v$ is the average sound velocity, $\rho_{M}$ is the mass density, $V$ is the system volume and $d$ is a coupling constant that depends on the electron-acoustic phonon coupling mechanism. Detailed expressions for the parameter $d$ can be found in Ref. [9]. The spin-flip transition rate $W$ is calculated from the Fermi Golden Rule.

\section{Results}

We show in the Fig. 1, the calculated spin inversion transition rates as a function of the lateral dot size $R$ for a InAs quantum dot. We have considered typical values for the external magnetic fields. From these results we can observe some interesting facts: i) the rates depend strongly with the magnetic field and can increases several order of magnitude when values of $B$ are swept from $0.5 \mathrm{~T}$ to $>1 \mathrm{~T}$. The magnitude of the rates increases when the dot size is increased and in the region $R>100 \AA$ depends weakly with the size. The magnitudes and behavior of the rates are compatible with the results obtained by Khaetskii and Nazarov [3] considering admixture coupling processes with acoustic phonons. Our results demonstrate that for narrow gap materials, the DP (dashed lines) and PE (solid lines) coupling presents comparable contributions to the spin relaxation process. Indeed, for large dots and large magnetic fields, the DP interaction becomes dominant.

This behavior is not observed in GaAs (see Fig. 2), where the PE coupling, in general, governs the relaxation process. An important aspect of our calculation is the dependence of the rates with the transition energy. In general, transition energy. In general, we obtain that $W \sim$ $\left(g \mu_{B} B\right)^{n}$, being $n$ an integer number that depends on the electron-phonon coupling process ( $n=7$ for DP and $n=5$ for $\mathrm{PE})$. This strong dependence with the transition energy and in consequence with the $g$-factor demands that this parameter should be determined taking in account the effects of

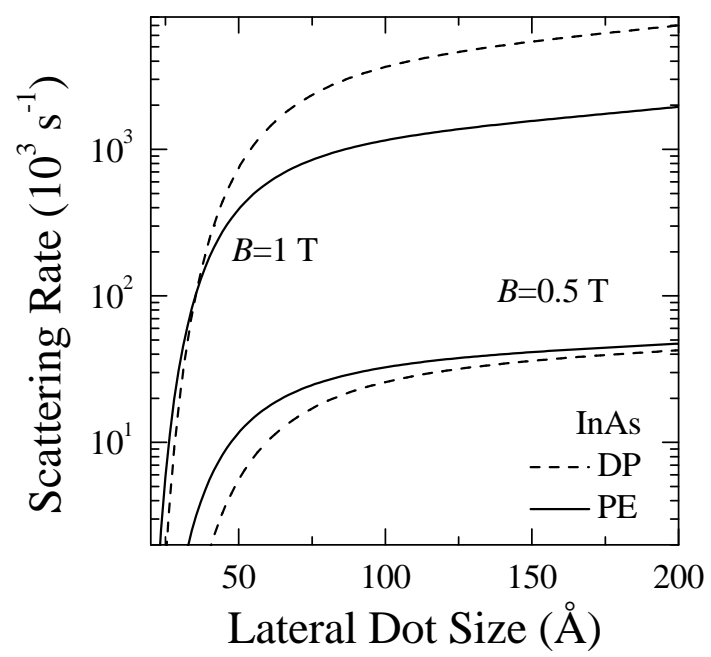

Figure 1. Spin-relaxation rates for an InAs quantum dot as a function of the lateral dot size $R$ for some values of $B$. DP and PE electron-phonon interactions are considered.

the quantum confinement. Experimental measurements of spin splitting in single InAs self-assembled quantum dots [11] reveal that the $g$-factor shows a clear dependence on the dot size. It increases with increasing energy gap between valence band electrons and conduction band holes, i.e. decreasing dot size. Values in the range of 0.77-1.29 were obtained for dots in strong confinement regime. These values differ strongly from the value of bulk InAs (-14.4). To include qualitatively the $g$-factor effects on our rate calculations we apply a Roth formula (2) based on a $\mathbf{k} \cdot \mathbf{p}$ model. Our results for InAs quantum dots, show that the effect of confinement on the $g$-factor produces significant variations in the relaxation rates. For instance, for DP mechanism, $B=1 \mathrm{~T}$ and $R=50 \AA$ we obtain $W \approx 10^{9}\left(\mathrm{~s}^{-1}\right)$ when $g=g_{\text {bulk }}$, if $g$ is given by (2), we obtain $W \approx 10^{8}\left(s^{-1}\right)$ The $g$-factor effects can be neglected for $R>150 \AA$. For GaAs case, the confinement does not produce important modifications in the $g$-factor and the scattering rates shown in the Fig. 2 reveal the negative character of the $g$ for the considered dot sizes.

Also, the Fig. 2 shows that the GaAs rates diminish abruptly when the dot size increases, for $R>100 \AA$ the relaxation times are up to seconds, and the rate depend weakly with the dot size. This behavior and rate magnitudes are similar to those obtained by Woods [4] considering other relaxation mechanisms mediated for acoustic phonons.

\section{Conclusion}

In conclusion, we have studied the spin relaxation of conduction electrons in InAs and GaAs parabolic quantum dots considering the phonon modulation of the spin-orbit interaction as a relaxation process. For quantum dots based on narrow-gap materials, we demonstrate that the effect of the $g$-factor should be necessarily included in the rate calculations and that the DP mechanism can be dominant, especially for large $B$. In general, for quantum dot sizes $>100 \AA$ we observe small size dependence of the rates in agreement with the experimental results of Gupta and Kikkawa. [12] 


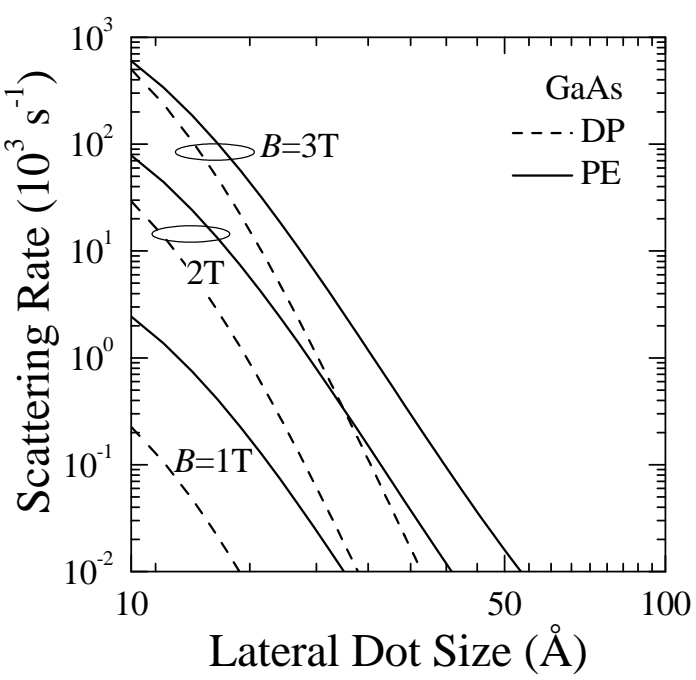

Figure 2. Same as Fig. 1 for GaAs quantum dots.

\section{Acknowledgments}

This work has been supported by Fundação de Amparo à Pesquisa do Estado de São Paulo (FAPESP) and by Conselho Nacional de Desenvolvimento Científico e Tecnológico (CNPq).

\section{References}

[1] W H Lau, J T Olesberg, and M E Flateé. Phys. Rev. B, 64:161301, 2001.

[2] A V Khaetskii and Y V Nazarov. Phys. Rev. B, 61:12636$12642,2000$.

[3] A V Khaetskii and Y V Nazarov. Phys. Rev. B, 64:125316, 2001.

[4] L. M. Woods, T. L. Reinecke, and Y. Lyanda-Geller. Phys. Rev. B, 66:161318(R), 2002.

[5] P. Hawrylak. Phys. Rev. B, 60:5597-5608, 1999.

[6] M H Weiler. Magnetooptical Properties of $\mathrm{Hg}_{1-x} \mathrm{Cd} \mathrm{T}_{x} \mathrm{Te} \mathrm{Al}$ loys, volume 16 of Semiconductor and Semimetals, chapter 3, 119-191, 1981.

[7] The higher-band contributions are assumed $F \approx 0$, and $N_{1} \approx 0$.

[8] S T Pavlov and Yu A. Firsov. Sov. Phys. Solid State, 7:21312140, 1966.

[9] S T Pavlov and Yu A Firsov. Sov. Phys. Solid State, 9:1394$1402,1967$.

[10] A A Sirenko, V I Belitsky, T Ruf, M Cardona, A I Ekimov, and C Trallero-Giner. Phys. Rev. B, 58:2077-2087, 1998.

[11] A S G Thornton, T Ihn, P C Main, L Eaves, and M Henini. Appl. Phys. Lett., 73:354-356, 1998.

[12] J A Gupta, D D Awschalom, X Peng, and P Alivisatos. Phys. Rev. B, 59(16):R10421-R10424, 1999. 